\title{
Neutrophil interactions with epithelial-expressed ICAM-1 enhances intestinal mucosal wound healing
}

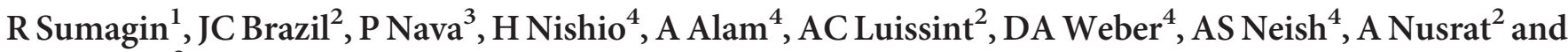 \\ CA Parkos ${ }^{2}$
}

A characteristic feature of gastrointestinal tract inflammatory disorders, such as inflammatory bowel disease, is polymorphonuclear neutrophil (PMN) transepithelial migration (TEM) and accumulation in the gut lumen. PMN accumulation within the intestinal mucosa contributes to tissue injury. Although epithelial infiltration by large numbers of PMNs results in mucosal injury, we found that PMN interactions with luminal epithelial membrane receptors may also play a role in wound healing. Intercellular adhesion molecule-1 (ICAM-1) is a PMN ligand that is upregulated on apical surfaces of intestinal epithelial cells under inflammatory conditions. In our study, increased expression of ICAM-1 resulted in enhanced PMN binding to the apical epithelium, which was associated with reduced PMN apoptosis. Following TEM, PMN adhesion to ICAM-1 resulted in activation of Akt and $\beta$-catenin signaling, increased epithelial-cell proliferation, and wound healing. Such responses were ICAM-1 dependent as engagement of epithelial ICAM-1 by antibody-mediated cross-linking yielded similar results. Furthermore, using an in-vivo biopsy-based, colonic-mucosalinjury model, we demonstrated epithelial ICAM-1 has an important role in activation of epithelial Akt and $\beta$-catenin signaling and wound healing. These findings suggest that post-migrated PMNs within the intestinal lumen can regulate epithelial homeostasis, thereby identifying ICAM-1 as a potential therapeutic target for promoting mucosal wound healing.

\section{INTRODUCTION}

Repeated injury of mucosal surfaces, particularly in the gastrointestinal tract, necessitate constant epithelial repair. Following injury, rapid resealing of the epithelial barrier is essential to prevent luminal bacteria and antigens from accessing the surrounding tissue and triggering inappropriate activation of innate and adaptive immune system components. Epithelial wound repair is accomplished by increased cell migration and proliferation. ${ }^{1,2}$ Both are complex processes that are regulated by many signaling molecules, including various growth factors and cytokines, ${ }^{3,4}$ which can act at basal and apical intestinal epithelial cell (IEC) membranes. In particular, $\beta$-catenin signaling has emerged as a key regulator of IEC proliferation and survival. ${ }^{3,5}$
Gastrointestinal disorders, such as inflammatory bowel diseases (IBDs), feature polymorphonuclear neutrophil (PMN) infiltration of intestinal mucosa and repeated epithelial injury. ${ }^{6,7}$ PMN migration across epithelial monolayers is often associated with barrier defects, ${ }^{8,9}$ epithelial injury, and crypt abscesses formation. ${ }^{10,11}$ However, as evident from recent work, PMNs may also play important temporal roles in resolution of inflammation and healing processes. For example, PMNs secrete lipid mediators, including lipoxins, resolvins, and protectins, that facilitate tissue healing. ${ }^{12,13}$ Furthermore, PMN migration across lung epithelial cells triggers transcriptional activation of $\beta$-catenin and its target genes, ${ }^{14,15}$ suggesting that PMNs, through interactions with IECs, can contribute to the regulation of epithelial cell proliferation.

${ }^{1}$ Department of Pathology, Feinberg School of Medicine, Northwestern University, Chicago, Illinois, USA. ${ }^{2}$ Department of Pathology, University of Michigan Medical School, Ann Arbor, Michigan, USA. ${ }^{3}$ Departamento de Fisiología, Biofísica y Neurociencias, Centro de Investigación y de Estudios Avanzados, Instituto Politécnico Nacional, Mexico, Mexico and ${ }^{4}$ Department of Pathology, Emory University School of Medicine, Atlanta, Georgia, USA. Correspondence: R Sumagin or CA Parkos (ronen.sumagin@northwestern.edu or cparkos@med.umich.edu) 
Inflammatory cues in the intestine also lead to increases in the expression of adhesive receptors at the apical epithelial membrane. Specifically, the apically expressed epithelial proteins CD44v6 and CD55 have both been shown to regulate PMN transepithelial migration (TEM). ${ }^{16,17}$ Another such epithelial adhesive ligand for migrating PMNs is Intercellular adhesion molecule-1 (ICAM-1). Its expression was found to facilitate PMN adhesion and retention at the apical epithelial membrane in inflamed intestines. ${ }^{18}$ Furthermore, ligation of ICAM-1 by migrating PMNs has been shown to signal cytoskeletal reorganization in both endothelial and epithelial cells, leading to alterations in barrier function. ${ }^{18-20}$ In inflamed vascular endothelial cells, specific cross-linking of ICAM-1 has been shown to induce activation of Akt signaling. ${ }^{21}$ In IECs, Akt acts upstream of $\beta$-catenin to induce signaling events that play key roles in regulating epithelial cell proliferation. . 22,23

In this study, we hypothesized that, following injury, PMNs that are recruited to sites of injury or inflammation and remain in contact with the apical epithelial membrane through binding to ICAM-1 can initiate reparative responses that are dependent on ICAM-1 signaling. Indeed, we demonstrated that PMN binding to or direct antibody $(\mathrm{Ab})$-mediated ligation of ICAM1 triggered activation of Akt and $\beta$-catenin signaling and promoted intestinal epithelial repair.

\section{RESULTS}

ICAM-1-dependent PMN adhesion to the apical IEC membrane results in delayed PMN apoptosis

Upregulation of ICAM-1 in IBD can lead to increased PMN adhesion and retention at the apical epithelial membrane. We recently showed, in an in-vitro assay modeling PMN TEM (a Transwell set-up ${ }^{17}$ ), that interferon gamma (IFN $\gamma$ )-induced upregulation of epithelial ICAM-1 significantly increased the number of apically associated PMNs following TEM. ${ }^{18}$ Here, we extend these observations to show that time-dependent increases in epithelial ICAM-1 expression following IFN $\gamma$ treatment $\left(100 \mathrm{U} \mathrm{ml}^{-1}\right.$, Figure 1a,b) coincide with timedependent increases in PMN adhesion (Figure 1c). This suggests that increased levels of ICAM-1 may indeed contribute to PMN accumulation in luminal spaces, as observed in IBD. Moreover, IFN $\gamma$-induced increases in PMN adhesion were specific to ICAM-1, as addition of ICAM-1 inhibitory Abs suppressed this PMN response (Figure 1c), but addition of immunoglobulin $\mathrm{G}$ (IgG) Abs as a control had no effect.

PMNs are typically considered to be short-lived cells; however, recent evidence suggests that PMN activation can delay PMN apoptosis. ${ }^{24,25}$ Inflammation of the intestinal mucosa features increasing numbers of PMNs in luminal spaces where they continue to interact with apical epithelial membranes. We thus hypothesized that PMN accumulation in inflamed mucosa could be partly due to delayed PMN apoptosis resulting from PMN activation during TEM and adhesion of post-migrated PMNs to luminal surfaces. Consistent with previous work, we found that PMN activation with $N$-formylmethionyl-leucyl-phenylalanine (fMLF; $100 \mathrm{~nm}$ ), or PMN chemotaxis toward a gradient of fMLF, significantly decreased the number of apoptotic cells after $10 \mathrm{~h}$ in culture, as compared with unstimulated PMNs in suspension $(27.8 \pm 3.2 \%$ and $26.8 \pm 2.2 \%$ vs. $42.7 \pm 3.4 \%$, respectively, Figure 1d). A greater reduction in the number of apoptotic PMNs was observed following TEM $(15.7 \pm 2.4 \%)$, consistent with increased PMN activation as evidenced by increased expression of CD11b (Supplementary Figure S1a online). Post-migrated PMN adhesion to epithelium further decreased the number of apoptotic PMNs (9.2 $\pm 1.4 \%$, Figure 1d, and representative flow diagrams of Annexin $\mathrm{V}$ - and propidium iodide-stained PMNs, Figure 1e). Interestingly, we found no significant difference in post-migrated PMN activation, assessed by expression of CD11b (Supplementary Figure S1a), between PMNs adhered to epithelium and those not adhered to epithelium, suggesting that continued PMN contact with epithelial monolayers may provide additional anti-apoptotic cues independent of cell activation. These findings imply that delayed apoptosis may contribute to PMN accumulation in inflamed mucosa. These observations further suggest that continued interactions of post-migrated PMNs with apical epithelial ligands might have profound, long-term effects on epithelial homeostasis.

\section{PMN engagement of ICAM-1 triggers activation of Akt and $\beta$-catenin signaling}

A prior study suggested that PMN migration across lung epithelial cells induces activation of $\beta$-catenin. ${ }^{14}$ Furthermore, in vascular endothelial cells, Ab-mediated cross-linking of ICAM-1 resulted in activation by increased phosphorylation of $\mathrm{Akt}^{21}$ As, in IECs, Akt-dependent activation of $\beta$-catenin signaling contributes to the regulation of IEC proliferation, 5,23 we investigated whether the binding of post-migrated PMNs to apically expressed ICAM-1 could trigger epithelial activation of Akt and $\beta$-catenin signaling. To this effect, PMNs were stimulated to migrate across epithelial monolayers. Transmigrated cells were collected and re-applied to the apical surface of new epithelial monolayers $\left(2.5 \times 10^{5} \mathrm{PMN}\right.$ per monolayer $)$ for $1 \mathrm{~h}$ with and without IFN $\gamma$ pretreatment. IFN $\gamma$ pretreatment was used to simulate inflammation and induce expression of ICAM-1. Consistent with results from previous studies, ${ }^{23}$ IFN $\gamma$ treatment enhanced phosphorylation of both Akt and $\beta$-catenin (Figure 2a). However, addition of post-migrated PMNs to IFN $\gamma$-stimulated IECs induced a further increase in Akt and $\beta$-catenin phosphorylation (Figure 2a). This increase was PMN-IEC contact dependent and was suppressed with inhibition of PMN adhesion using an inhibitory Ab to CD11b (CBRM1/29, $20 \mu \mathrm{g} \mathrm{ml}^{-1}$ ). Confirming ICAM-1's role in the observed PMN-mediated effects, Ab-mediated cross-linking of ICAM- 1 on the apical surface of IFN $\gamma$-stimulated IECs induced a robust increase in Akt and $\beta$-catenin phosphorylation, consistent with that observed in PMN adhesion; but use of a control protein (MHC-1) resulted in no such increase (Figure 2b). Consistent with previous findings, ${ }^{23}$ IFN $\gamma$ treatment induced upregulation of the total level of Akt without affecting the expression of $\beta$-catenin. Application of post-migrated PMNs or anti-ICAM-1 cross-linking Abs to 
a

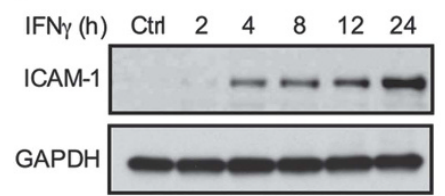

d

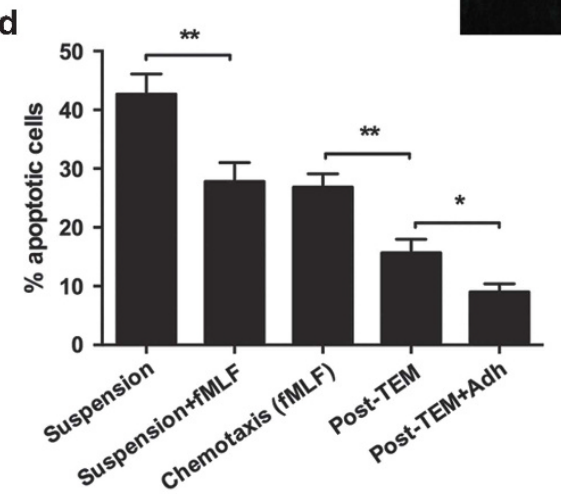

b

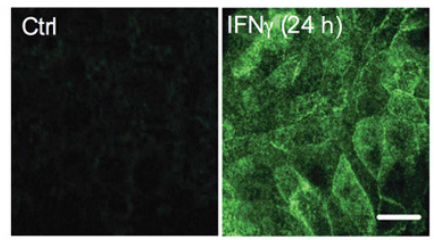

C
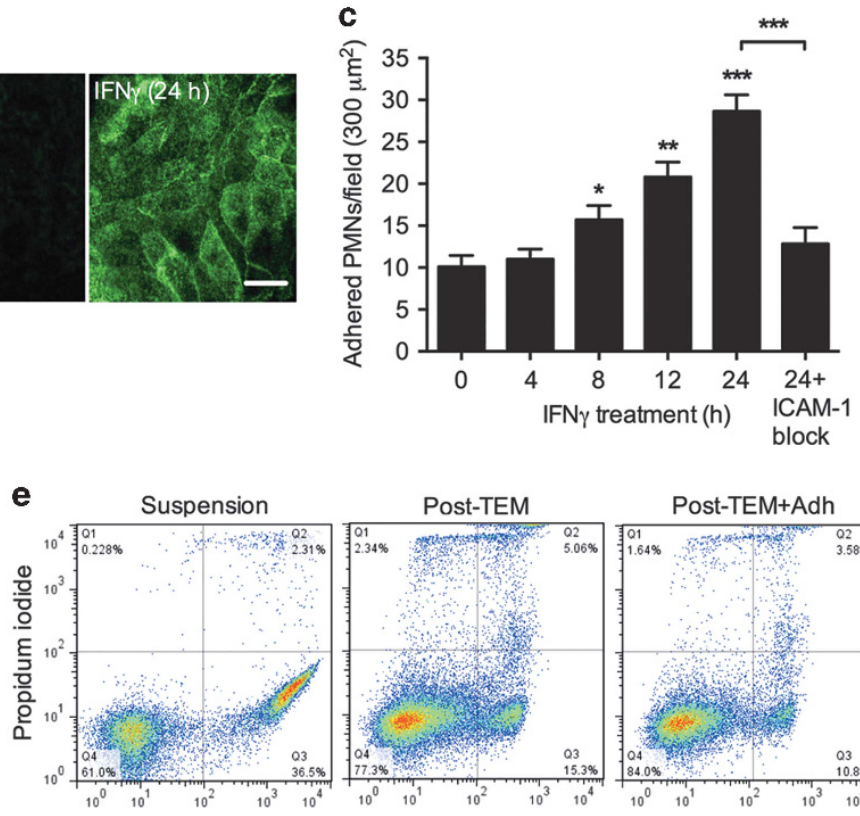

Post-TEM

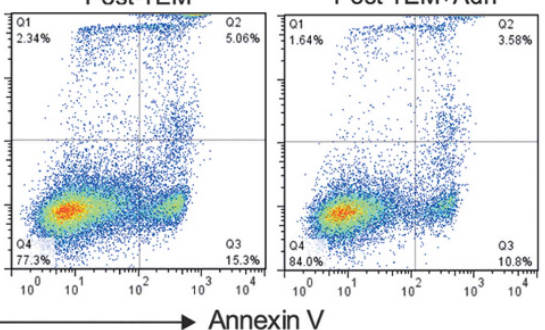

Figure 1 Intercellular adhesion molecule-1 (ICAM-1)-dependent increases in polymorphonuclear neutrophil (PMN) adhesion to the apical intestinal epithelial cell (IEC) membrane renders PMNs less susceptible to apoptosis. (a) Western blot analyses reveal time-dependent upregulation of ICAM-1 in confluent T84 IECs following treatment with interferon gamma (IFN $\gamma ; 100 \mathrm{U} \mathrm{ml}^{-1}$ ). Equal protein loading was confirmed using house-keeping gene glyceraladehyde-3- phosphate dehydrogenase (GAPDH). (b) Induction of ICAM-1 expression was confirmed by immunofluorescence staining. The scale bar represents $20 \mu \mathrm{m}$. For $\mathbf{a}$ and $\mathbf{b}$, the images are representative of five independent experiments. (c) PMNs following transepithelial migration (TEM) were collected and introduced to the apical membranes of T84 IECs $\left(2.5 \times 10^{5}\right.$ cells per well in $\left.\mathrm{H}+, 1 \mathrm{~h}, 37^{\circ} \mathrm{C}\right)$ that were pretreated with IFN $\gamma$, times as indicated, in the presence or absence of anti-ICAM-1 inhibitory Ab $\left(20 \mu \mathrm{g} \mathrm{ml}^{-1}\right)$. The number of adherent PMNs per high-power field was quantified. $N=4$ independent experiments with two technical replicates for each condition; ${ }^{*} P<0.05,{ }^{* *} P<0.01$, ${ }^{* * *} P<0.001$; different from $t=0$, analysis of variance (ANOVA) with Newman-Keuls multiple comparison test. (d,e) The number of apoptotic PMNs following $10 \mathrm{~h}$ culture under the conditions indicated in the figure was measured with Anexin- $V$ and propidium iodide staining using flow cytometry. PMNs were distinguished from epithelial cells by staining and gating on CD11b. (d) Quantification and (e) flow diagrams representing four independent experiments show percent apoptotic PMN under the defined conditions. All Annexin V-positive, propidium iodide-negative cells were considered as apoptotic. PMN adhesion to apical epithelial membrane renders them less susceptible to apoptosis. $N=4$ independent experiments; ${ }^{*} P<0.05$, ${ }^{* \star} P<0.01$; ANOVA with NewmanKeuls multiple comparison test. Ctrl, control.

non-stimulated monolayers, without ICAM-1 expression, had no significant effect on Akt and $\beta$-catenin signaling. To further test the effects of ICAM-1-dependent signaling on Akt and $\beta$-catenin activation in the absence of potential effects of cytokine treatment, $\mathrm{CHO}-\mathrm{K} 1$ cells, which lack endogenous expression of ICAM-1 but can activate Akt and $\beta$-catenin signaling, were transfected to stably express ICAM-1. Intriguingly, overexpression of ICAM-1 in CHO-K1 cells was sufficient to induce increases in Akt and $\beta$-catenin phosphorylation (Supplementary Figure S2). Consistent with findings in IECs, these increases were further potentiated following ICAM-1 cross-linking.

Next, we used the TOPFlash reporter assay to examine $\beta$-catenin transcriptional activation following ICAM-1 ligation. We demonstrated that both PMN adhesion and Ab-mediated engagement of ICAM-1 triggered significant increases in $\beta$-catenin transcriptional activity (Figure $2 \mathrm{c}, \mathbf{d}$ ). These increases required pre-stimulation of the epithelium with IFN $\gamma$, consistent with IFN $\gamma$-mediated induction of ICAM-1 expression. In both cases, activation of T-cell factor/lymphoid enhancer factor (TCF/LEF) reporter TOPFlash was observed; but no such activation was observed in a control with mutated
TCF/LEF-binding sites. PMN-adhesion-induced $\beta$-catenin transactivation was contact dependent and was suppressed in the presence of anti-CD11b inhibitory Abs. Finally, inhibition of Akt activity (Akt inhibitor VIII, $10 \mu \mathrm{M}, 30 \mathrm{~min}$ ) before addition of either PMNs or anti-ICAM-1 cross-linking Abs prevented TOPFlash activation. This finding was consistent with Akt activation being upstream of $\beta$-catenin and playing a key role in $\beta$-catenin activation.

\section{PMN engagement of ICAM-1 promotes IEC proliferation}

As Akt and $\beta$-catenin have an important role in the regulation of IEC proliferation, ${ }^{5}$ we next examined whether ICAM-1dependent activation of Akt and $\beta$-catenin signaling subsequently resulted in enhanced IEC proliferation. Using similar approaches as described above, post-migrated PMNs or anti-ICAM-1 cross-linking Abs were applied to IECs, with and without IFN $\gamma$ pretreatment, and IEC proliferation was assessed using 5-ethyl-2'-deoxyuridine (EdU) incorporation. Although IFN $\gamma$ treatment alone had no significant effect on cell proliferation, $\mathrm{PMN}$ adhesion and direct $\mathrm{Ab}$ engagement of ICAM-1 significantly increased IEC proliferation (Figure 3a,b,d). These increases in IEC proliferation were 

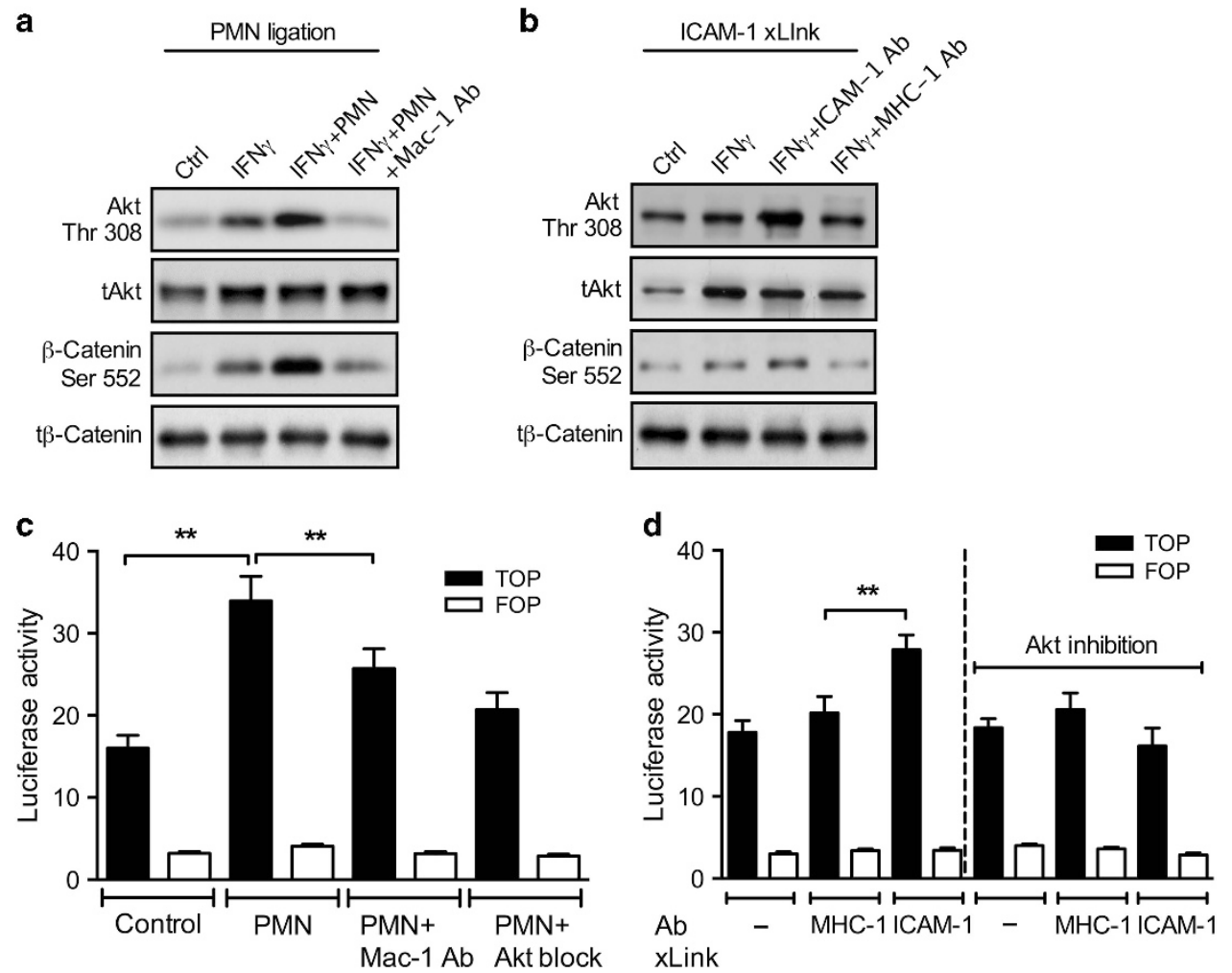

Figure 2 Polymorphonuclear neutrophil (PMN) engagement of intercellular adhesion molecule-1 (ICAM-1) triggers activation of Akt and $\beta$-catenin signaling. Control (Ctrl) and interferon gamma (IFN $\gamma$ )-stimulated T84 intestinal epithelial cells (IECs) grown on permeable supports were incubated with either PMN that migrated across T84 IECs $\left(2.5 \times 10^{5}\right.$ cells per well) (a) or with anti-ICAM- 1 cross-linking antibodies (Abs; to specifically cluster ICAM-1) (b). The effects of PMN apical adhesion and ICAM-1 cross-linking on Akt and $\beta$-catenin activation (increased phosphorylation) were assessed using western blot analysis. All western blots are representative of $3-5$ independent experiments. (c,d) $\beta$-Catenin transcriptional activity was analyzed using the TopFlash luciferase reporter assay. SW480 IECs were transfected with the $\beta$-catenin reporter (TopFlash; full bars) or its mutated form (FopFlash; empty bars) $72 \mathrm{~h}$ before the addition of PMN or Ab-mediated ICAM-1 cross-linking. Where indicated, inhibitory Abs to CD11b (Mac-1, 20 $\left.\mu \mathrm{g} \mathrm{ml}{ }^{-1}\right)$ were introduced in combination with PMNs. IECs were incubated with Akt inhibitor (Inhibitor VIII, $10 \mu \mathrm{M}$ ) 30 min before addition of either PMNs or anti-ICAM-1 cross-linking Abs. PMN ligation or specific Ab-mediated engagement of ICAM-1 triggers activation of Akt and $\beta$-catenin signaling. $N=3$ independent experiments; ${ }^{*} P<0.01$; analysis of variance with Newman-Keuls multiple comparison test.

consistent with the observed Akt and $\beta$-catenin activation and required IFN $\gamma$ stimulation. Furthermore, confirming Akt's role in the observed responses, addition of Akt inhibitor (Akt Inhibitor VIII, $10 \mu \mathrm{M}, 30 \mathrm{~min}$ ) before application of PMNs or ICAM-1-cross-linking protocols suppressed an increase in IEC proliferation (Figure 3c).

ICAM-1-dependent increases in cell proliferation promote IEC wound healing in-vitro

Increased cell proliferation is a major component of epithelial wound healing. ${ }^{26,27}$ Given our observations that ICAM-1dependent activation of $\beta$-catenin resulted in increased IEC proliferation, we next hypothesized that ligation of ICAM-1 and initiation of ICAM-1-dependent signaling would promote IEC wound healing. To test this hypothesis, we first examined the effect of ICAM-1 cross-linking on cellular proliferation at the wound edge. As expected, application of anti-ICAM-1 cross-linking Abs to IFN $\gamma$-treated IEC monolayers induced clustering of ICAM-1, resulting in a significant increase in the number of proliferating cells at the wound edge; but application of MHC-1 had no such effect (Figure 4a,b).
Although inducing expression of ICAM-1, IFN $\gamma$ treatment of T84 IECs also inhibits wound closure. ${ }^{28}$ Thus, we investigated whether Ab-mediated cross-linking of ICAM-1 and induction of ICAM-1-dependent signaling would suppress IFN $\gamma$-mediated inhibition of wound healing. In these experiments, anti-ICAM-1 cross-linking Abs were introduced to scratch-wounded IEC monolayers $24 \mathrm{~h}$ following IFN $\gamma$ treatment; and the effects of ICAM-1 cross-linking on IEC wound closure were quantified. As expected, IFN $\gamma$ treatment markedly delayed wound closure $48 \mathrm{~h}$ post-injury (Figure $4 \mathrm{c}, \mathbf{d}$ ). However, addition of anti-ICAM-1 cross-linking Abs reversed the IFN $\gamma$-dependent inhibitory phenotype. To confirm the role of ICAM-1 signaling in IEC wound healing independently of IFN $\gamma$-mediated effects, similar experiments were performed with Caco-2 IECs that constitutively express ICAM- $1{ }^{29} \mathrm{We}$ observed that scratch wounding had no effect on expression of ICAM-A (Supplementary Figure S4a). However, consistent with observations in T84 IECs, introduction of anti-ICAM-1 cross-linking Abs to scratch-wounded Caco-2 monolayers immediately after wounding significantly enhanced wound closure both at 24 and $48 \mathrm{~h}$ (Supplementary Figure S4b). To 

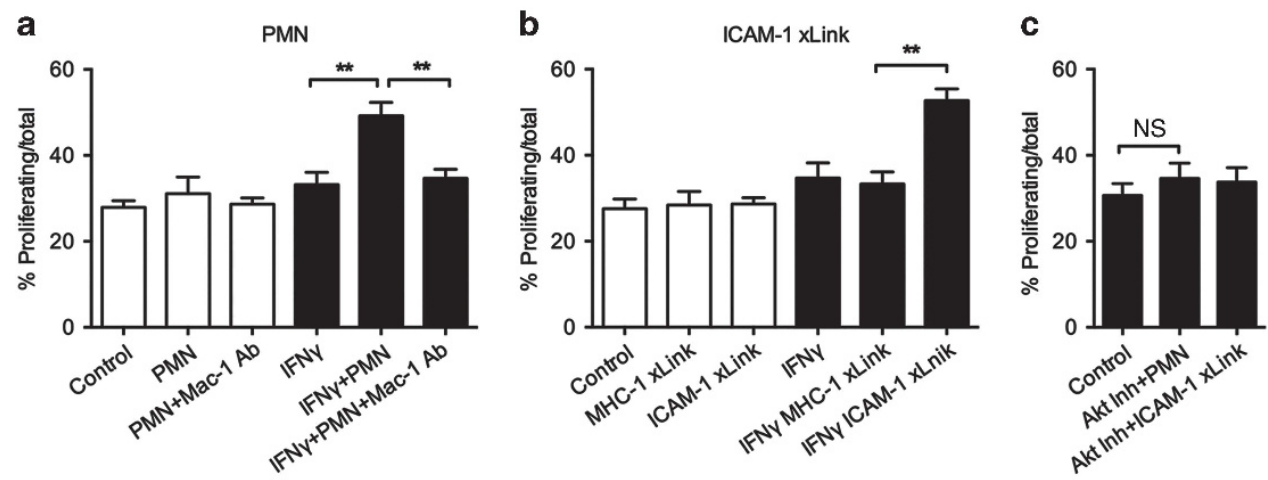

d
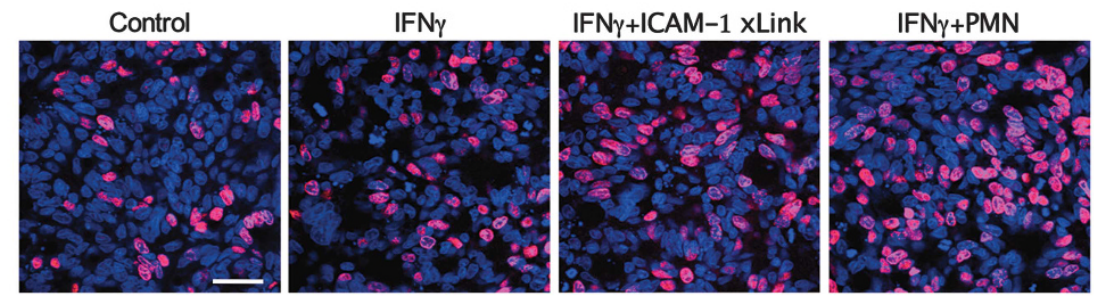

Figure 3 Ligation of intercellular adhesion molecule-1 (ICAM-1) promotes intestinal epithelial cell (IEC) proliferation. (a-c) Unstimulated (white bars) or interferon gamma (IFN $\gamma$ )-stimulated (black bars, $100 \mathrm{U} \mathrm{ml}^{-1}, 24 \mathrm{~h}$ ) T84 IECs (to induce expression of ICAM-1) were subjected to (a) Polymorphonuclear neutrophils (PMNs) that were collected following transepithelial migration (TEM; $2.5 \times 10^{5}$ cells per well), (b) anti-ICAM-1 or anti-MHC-1 (a control protein) cross-linking antibodies (Abs). (c) IFN $\gamma$-stimulated IECs were incubated with Akt inhibitor (Inhibitor VIII, $10 \mu \mathrm{M}) 30$ min before addition of either PMNs or anti-ICAM-1 cross-linking Abs. The effects of ICAM- 1 ligation by adhered PMN or Ab-mediated cross-linking of ICAM-1 on IEC proliferation were assessed using 5-ethyl-2'-deoxyuridine incorporation and confocal immunofluorescence imaging. Data were shown as percent proliferating cells out of total cells. For all data sets, $N=3$ independent experiments with three technical replicates for each condition; ${ }^{* *} P<0.01$; analysis of variance with Newman-Keuls multiple comparison test. (d) Fluorescence images, representative of three independent experiments, show increased number of proliferating cells (red, non-proliferating cells are shown in blue) following Ab and PMN cross-linking of ICAM-1. The scale bar is $10 \mu \mathrm{m}$. NS, not significant.

further confirm specificity of these effects to ICAM-1, crosslinking of CD55, which localizes to apical epithelial membranes and mediates PMN adhesive interactions, ${ }^{16,18}$ had no significant effect of IEC wound closure (Supplementary Figure S4b). We next examined whether binding of post-migrated PMNs to ICAM-1 at the wound edge would similarly promote wound healing. In these experiments, post-migrated PMNs were added to scratch-wounded IECs $24 \mathrm{~h}$ following IFN $\gamma$ treatment; and closure of the wounds in the presence of PMNs was quantified. Consistent with the results of $\mathrm{Ab}$-mediated cross-linking of ICAM-1, PMN binding to IEC monolayers suppressed IFN $\gamma$ mediated inhibition on wound closure (Figure 5a). Inhibition of CD11b-dependent PMN adhesion to IECs using inhibitory Abs prevented the rescue effect. Furthermore, application of anti-ICAM-1 cross-linking Abs or addition of post-migrated PMNs to unstimulated T84 IEC monolayers without ICAM-1 expression had no significant effect on IEC wound healing (Supplementary Figure S1b). Finally, the effect of postmigrated PMN binding to ICAM-1 on IEC wound healing was confirmed in Caco-2 cells. PMN binding to ICAM-1 on Caco-2 significantly enhanced wound closure independently of IFN $\gamma$ (Supplementary Figure S4c). Inhibition of CD11b significantly decreased PMN adhesion (not shown) to IECs and prevented the increase in IEC wound closure. Addition of CD11a, another PMN adhesion receptor that is known to bind ICAM $-1^{30}$ had only minor, non-significant effects on IEC wound closure (Supplementary Figure S4c). These findings may be explained by the much higher ratio of expressed CD11b to CD11a molecules on the activated PMN surface, which is greater than 5 to $1 .{ }^{31}$ Consistent with this finding, both PMN adhesion to and migration across IECs has been previously shown to be a CD11a-independent, CD11b-dependent process. ${ }^{32}$ Together, these experiments suggest that CD11b and ICAM-1 binding interactions have a predominant role in mediating the observed effects of ICAM-1 ligation on wound closure.

To confirm that ICAM-1-dependent rescue of IEC wound healing was due to enhanced IEC proliferation, scratchwounded IECs were pretreated with L-mimosine before $\mathrm{Ab}$ cross-linking or post-migrated PMN ligation of ICAM-1. L-Mimosine inhibits cell proliferation by arresting cells in late G1 phase. ${ }^{2,33}$ As expected, L-mimosine treatment significantly decreased cell proliferation, resulting in delayed wound closure (Figure 5b). Importantly, following L-mimosine treatment, neither application of anti-ICAM-1 cross-linking Abs nor binding of post-migrated PMNs was able to suppress the IFN $\gamma$-induced inhibition of wound healing.

\section{ICAM-1 expression and function regulate healing of mucosal wounds in-vivo}

We next examined the role of ICAM-1 in epithelial wound healing in-vivo, using a colonic-mucosal, biopsy-based-wound model. ${ }^{11,34,35}$ In this setup, superficial mucosal wounds were introduced to colons of wild-type (WT) and ICAM-1-deficient 


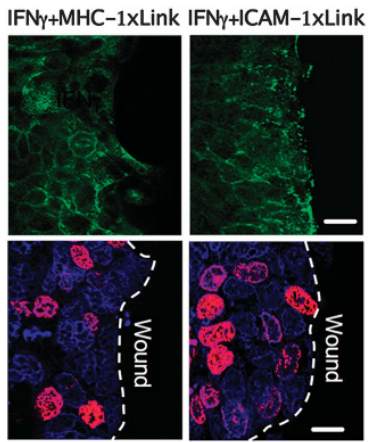

b

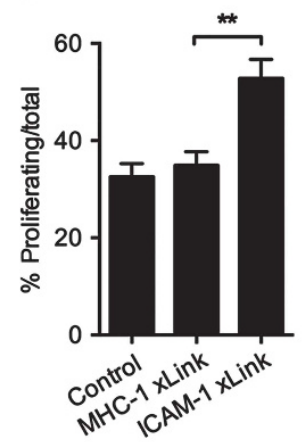

C

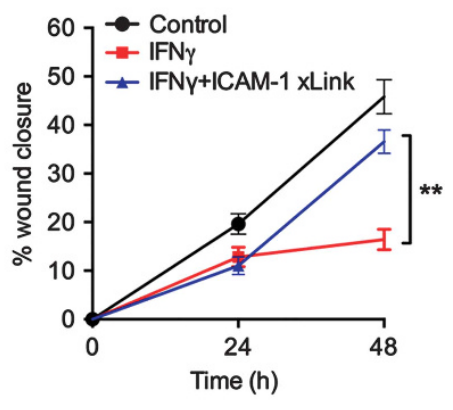

d

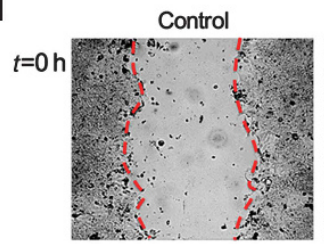

$\mathrm{IFN} \gamma$

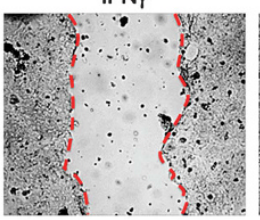

IFN $\gamma+$ ICAM-1 $x$ Link

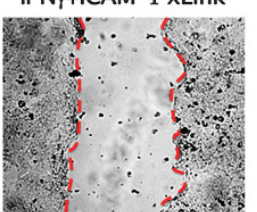

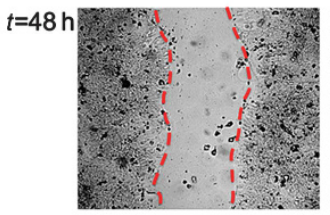
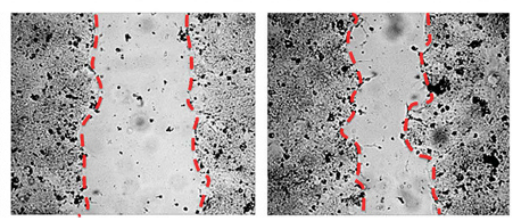

Figure 4 Intercellular adhesion molecule-1 (ICAM-1)-dependent increases in cell proliferation promote intestinal epithelial cell (IEC) wound healing in vitro. Scratch-wounded T84 IEC monolayers were stimulated with interferon gamma (IFN $\gamma ; 100 \mathrm{U} \mathrm{ml}^{-1}$ ) and were subjected to anti-ICAM-1 or antiMHC-1 cross-linking antibodies (Abs) $24 \mathrm{~h}$ later. (a, upper panels) Fluorescence mages representative of three independent experiments show wounded IEC monolayers that were immunofluorescently stained for ICAM-1. Clustering of ICAM-1 at wound edges following application of anti-ICAM-1, but not MHC-1 cross-linking Abs were observed. The scale bar represents $20 \mu \mathrm{m}$. (a, bottom panels) IEC proliferation was examined at wound edges $(50-\mu \mathrm{m}-$ width regions) following ICAM-1 cross-linking. Anti-ICAM-1 cross-linking Abs, but not anti-MHC-1 Abs, enhanced cell proliferation, which was quantified and is represented in $\mathbf{b} . N=3$ independent experiments with three technical replicates for each condition; ${ }^{* \star} P<0.01$, analysis of variance with NewmanKeuls multiple comparison test. (c) Wound closure was measured over $48 \mathrm{~h}$ in unstimulated (control), IFN $\gamma$-stimulated IEC monolayers, and IFN $\gamma$ stimulated IECs that were subjected to anti-ICAM-1 cross-linking Abs at $24 \mathrm{~h}$. $N=3$ independent experiments with three technical replicates for each condition; ${ }^{* *} P<0.01$; Student's $t$-test. (d) Images representative of three independent experiments show delayed wound healing in IFN $\gamma$-treated IECs and the rescuing effects of anti-ICAM-1 cross-linking Abs.
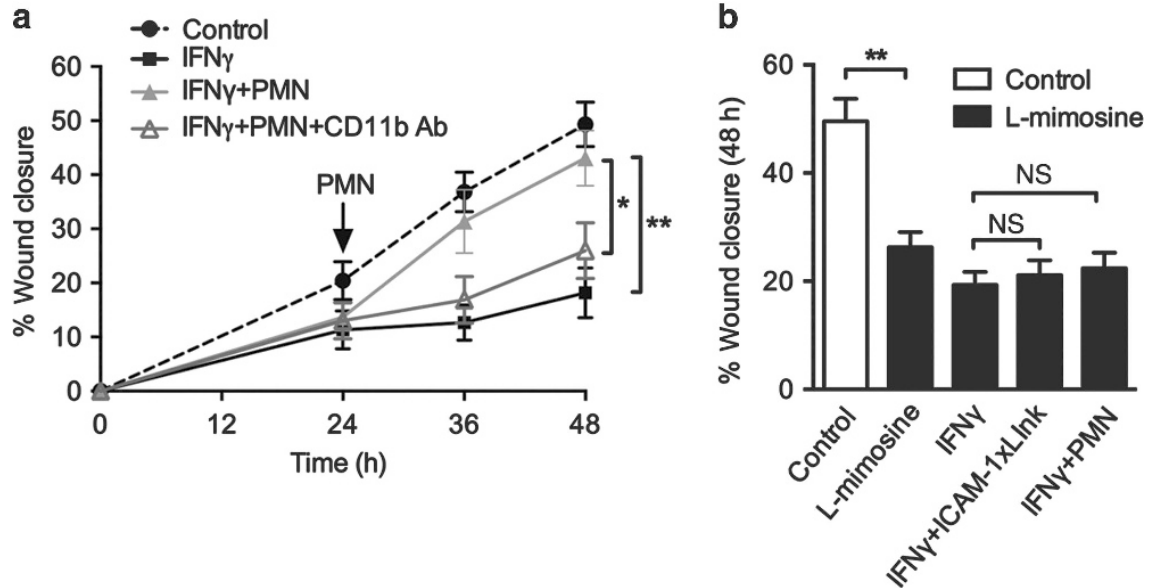

Figure 5 Adhesion of post-migrated polymorphonuclear neutrophils (PMNs) to apically expressed intercellular adhesion molecule-1 (ICAM-1) promotes intestinal epithelial cell (IEC) wound healing in vitro. (a) Confluent, unstimulated (control) T84 IEC monolayers were scratch wounded, and the healing process was monitored over $48 \mathrm{~h}$. When specified, IECs were treated with interferon gamma (IFN $\gamma ; 100 \mathrm{U} \mathrm{ml}^{-1}$ ) immediately following wounding and, at $24 \mathrm{~h}$ post-treatment, were incubated with post-migrated $\mathrm{PMNs}\left(2.5 \times 10^{5}\right.$ cells per well) in the presence or absence of CD11b inhibitory Ab (Mac-1, $20 \mu \mathrm{g} \mathrm{ml}^{-1}$ ). Consistent with Ab-ligation of ICAM-1, PMN adhesion to ICAM-1 reversed the IFN $\gamma$-induced inhibition of wound closure. PMN effects were $\mathrm{CD} 11 \mathrm{~b}$ dependent and were prevented in the presence of anti-CD11b inhibitory $\mathrm{Ab} . N=3$ independent experiments with three technical replicates for each condition; ${ }^{*} P<0.05,{ }^{* *} P<0.01$; ANOVA with Newman-Keuls multiple comparison test. (b) Unstimulated or IFN $\gamma$-treated IECs were incubated with L-mimosine $(100 \mu \mathrm{M}$, a potent inhibitor of cell proliferation) $12 \mathrm{~h}$ before addition of either PMNs or anti-ICAM-1 cross-linking Abs. Inhibition of cell proliferation using L-mimosine prevented ICAM-1 ligation-dependent suppression of the IFN $\gamma$ effect. $N=3$ independent experiments with three technical replicates for each condition; ${ }^{\star \star} P<0.01$, not significant (NS): $P>0.05$; ANOVA with Newman-Keuls multiple comparison test. 
(ICAM-1ko) mice. Immunofluorescence and western blot analyses of extracted mucosal wounds revealed increased expression of ICAM-1 by IECs (Supplementary Figure S3a,b). Mucosal wound healing was then monitored 2 and 4 days post wounding by endoscopic imaging. A significant, nearly threefold, delay in wound closure was observed in ICAM-1ko mice as compared with WT mice (Figure 6a,b). A delay in re-epithelialization of colonic mucosa was also evident in immunofluorescence labeling of frozen tissue sections from colonic mucosal wounds (day 4 after wounding, Figure 6c). Given evidence of ICAM-1-dependent, Akt/ $\beta$-catenin-driven increases in wound closure in cultured IECs (Figure 3), we next examined whether delayed wound closure in ICAM-1ko mice was due to decreased activation of Akt and $\beta$-catenin signaling, resulting in decreased epithelial cell proliferation. Cell proliferation was assessed by EdU incorporation assay and revealed a significant decrease in the number of proliferating cells in colonic crypts adjacent to wound edges $(500 \mu \mathrm{m})$ in ICAM-1ko compared with WT mice $(26.2 \pm 2.1$ vs. $37.6 \pm 1.6$ cells per villus, Figure $\mathbf{6 c}, \mathbf{d}$ ). Consistent with observations in human IECs, western blot analysis of WT mice solubilized colonic mucosa revealed a robust increase in phosphorylation of both Akt and $\beta$-catenin in healing wounds, as compared with non-wounded tissue (Figure 6e). Protein phosphorylation levels peaked at day 2 post wounding and subsided by day 4 when the healing process was nearly complete.

Unlike in WT mice, no increases in the levels of phosphorylated Akt and $\beta$-catenin were observed in mucosal wounds from ICAM-1ko mice (Figure 6e), implicating ICAM1 in Akt and $\beta$-catenin activation and further demonstrating that epithelial cell proliferation is dependent on $\beta$-catenin signaling in vivo. Further immunofluorescence analysis revealed infiltration of the wounded colonic mucosa by $\mathrm{CD}_{11 \mathrm{~b}}{ }^{+}$leukocytes in both WT and ICAM-1ko mice (Figure 6c). These observations confirm that the immune cells were present in ICAM-1ko mice to ligate ICAM-1 and suggest that the absence of activation of Akt and $\beta$-catenin signaling and the observed delay in wound closure were due to lack of ICAM-1 on enterocytes in these mice. A representative piece of a distal colon that was extracted at day 2 post injury and that was used for collection of wounded and non-wounded mucosal tissue is shown in Figure $6 \mathbf{f}$. a
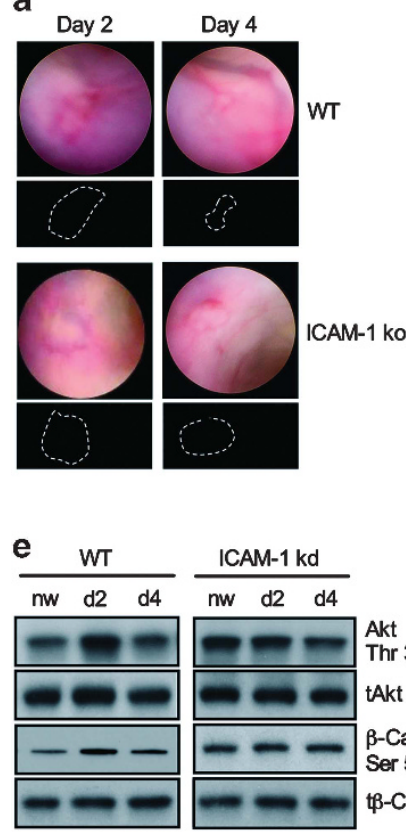
ICAM-1 kd
nw d2 d4

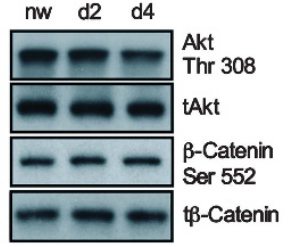

b

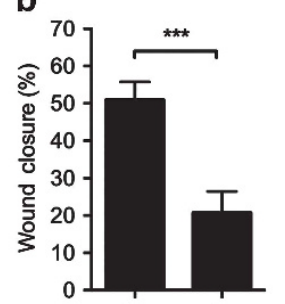

C
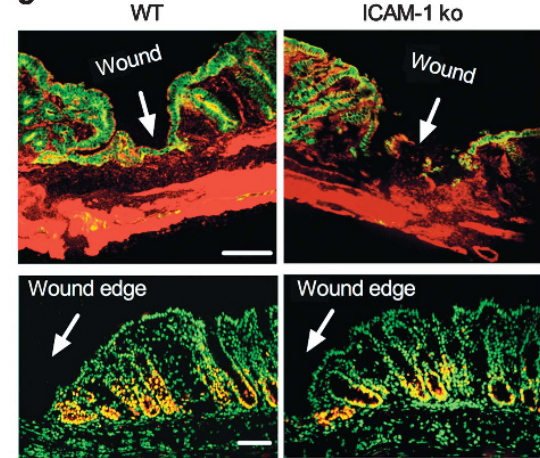

wit

d 50

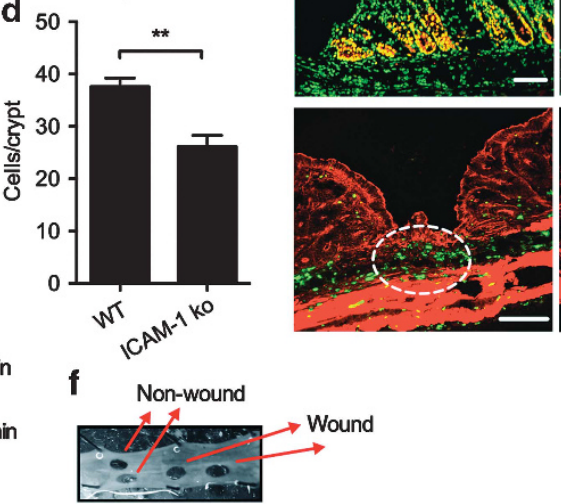

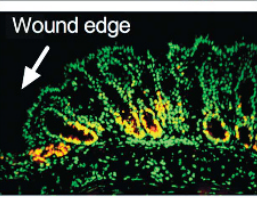

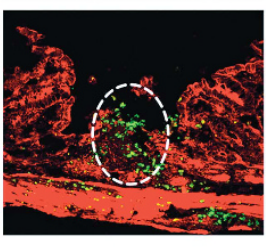

Figure 6 Intercellular adhesion molecule-1 (ICAM-1) expression in mucosal epithelium is essential for proper healing of mucosal wounds in vivo. Superficial wounds were introduced to mice colons in vivo using colonoscopic-biopsy-based technique, and wound healing was monitored and quantified using colonoscopic imaging. (a) The images depicted are representative of at least 36 wounds that were analyzed per condition; show colonic mucosal wounds at days 2 and 4 post injury in wild-type (WT) and ICAM-1 ko mice. (b) Day 4 post injury areas of mucosal wounds were quantified. ICAM-1 exhibited significant delay in wound closure. $N=2$ independent experiments; 6 mice per group; $3-4$ wounds per mouse ${ }^{* * *} P<0.001$; Student's $t$-test. (c, upper panels) Colonic mucosal wounds were extracted from WT and ICAM-1ko mice at day 2 following wounding and frozen in optimum cutting temperature solution. Eight-micrometer sections were stained for actin (red) and $\beta$-catenin (green) to visualize epithelial cells. Fluorescence images clearly show impaired re-epithelialization of wounded regions in the absence of functional ICAM-1. (c, middle panels) Proliferation of wound-edge epithelial cells in vivo was examined using 5-ethyl-2'-deoxyuridine incorporation assay. Fluorescence images and quantification depicted in $\mathbf{d}$ show decreased number of proliferating cells (yellow) in ICAM-1 ko mice. (c, bottom panels) Fluorescence images show CD11b-positive cells infiltrating the mucosal wound areas in both WT and ICAM-1ko mice outlined by dash-circled areas. All images in c, are representative of at least 18 wounds. Scale bars represent $200 \mu \mathrm{m}$. ${ }^{* *} P<0.01$; Student's $t$-test. (e) Wounded tissue, days 2 and 4 post injury, and equivalent non-wounded tissue were extracted using punch biopsy. Akt and $\beta$-catenin activation (increased phosphorylation) during the healing process was assessed using western blot analysis. Blots represent three independent experiments. (f) The image depicts punch-biopsy wounded and non-wounded regions of colon. 
Finally, to substantiate our findings and confirm the specific role of epithelial ICAM-1 in promoting mucosal healing, we assessed the effect of Ab-mediated cross-linking of ICAM-1 in vivo, in biopsy-generated mucosal wounds. In these experiments, immune complexes were generated by incubating anti-ICAM-1 Ab or control IgG with anti-Fc polyclonal Abs at a molecular ratio of 1:2, respectively (Supplementary Figure S3c). These complexes were injected into mucosal wounds $24 \mathrm{~h}$ post wounding using endoscopic procedure (Figure 7a). The effects of ICAM-1 and control IgG ligation by immune complexes on mucosal wound closure and epithelial cell proliferation were examined at day 4 post wounding. As shown in Figure 7b, injection of anti-ICAM-1 cross-linking, but not control IgG, complexes resulted in significantly enhanced wound closure, confirming ICAM-1-dependent signaling has a role in mucosal healing. Furthermore, immunofluorescence staining of harvested mucosal wounds revealed increased re-epithelialization of colonic mucosa and enhanced proliferation of wound edge epithelial cells following injection of anti-ICAM-1, but not control IgG, complexes (Figure $7 \mathrm{c}, \mathbf{d}$ ).
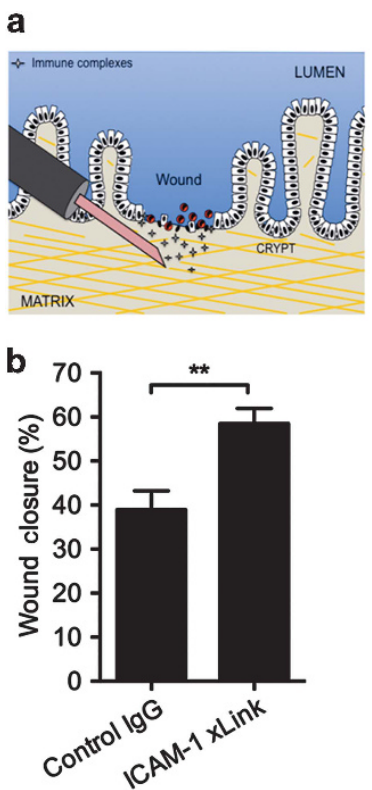

Figure 7 Specific engagement of epithelial intercellular adhesion molecule-1 (ICAM-1) promotes mucosal healing in vivo. (a) Cartoon depicting injection of immune complexes directly into the wound. (b) Superficial wounds were introduced to mice colons in-vivo using colonoscopic-biopsy-based technique. Twenty-four hours later, epithelial ICAM-1 was cross-linked via injection of anti-ICAM-1 or control immunoglobulin $\mathrm{G}(\mathrm{IgG})$ immune complexes into the wounds (shown in a). Mucosal wounds were visualized and measured using colonoscopic imaging at day 4 post wounding. At least 18 wounds from 6 mice per condition were analyzed. ${ }^{*} P<0.01$; Student's $t$-test. (c) Colonic mucosal wounds were extracted and optimum cutting temperature solution frozen. Eight-micrometer sections were stained for E-cadherin (green) and Ki67 (red) to visualize total and proliferating epithelial cells at wound edge. Fluorescence mages representative of 18 wounds show enhanced reepithelialization of wounded regions following injection of ICAM-1 crosslinking (bottom panel) but not following injection of IgG control (upper panel) immune complexes. To-Pro-3 iodide was used to visualize cell nuclei.

\section{DISCUSSION}

PMNs can act as a "double-edged sword" both by inflicting tissue damage and by providing protection against pathogens and promoting resolution of inflammation. ${ }^{36}$ In mucosal tissues and particularly the gastrointestinal tract, which is continually exposed to various bacterial pathogens, rapid mobilization of PMNs toward sites of infection is critical for efficient induction of successful adaptive immune responses and pathogen clearance. ${ }^{37}$ However, aberrant PMN infiltration and accumulation in mucosal tissues is often associated with barrier defects and injury. ${ }^{6,10,38}$ Our current findings add a contextual- and time-dependent dimension to PMN TEM, suggesting that, although infiltration of the epithelium by great numbers of PMNs as seen in active IBD may indeed be damaging to the surrounding tissue, PMNs in the intestinal lumen that are retained at the apical membrane may positively influence epithelial function and initiate epithelial repair.

Tissue damage associated with PMN migration across epithelial monolayers is typically attributed to PMN-granule-derived soluble mediators including elastase, cathepsins, and matrix metalloproteinases. ${ }^{10,39-41}$ These proteinases are well known for their ability to post-translationally modify soluble, membrane-bound and extracellular matrix proteins to promote their rapid delivery or inactivation. In addition to granule-contained proteinases, PMN migration across epithelial layers can also shed membrane bound protein ectodomains that can bind to epithelial ligands and affect epithelial function. For example, binding of junctional adhesion molecule-like protein (JAML), released from PMN during TEM, to coxsackie and adenovirus receptor at cell junctions was found to inhibit epithelial cell proliferation and wound healing. ${ }^{11}$ Both degranulation and shedding of surface proteins are dependent on PMN activation and can be induced by stimulation with various cytokines. ${ }^{11,42}$ PMN activation and degranulation are significantly potentiated during migration across epithelial monolayers. ${ }^{11,43}$ As a result, PMNs that reach luminal spaces are significantly depleted of some of these harmful mediators. Specifically, when expression of JAML was examined on PMNs before and after TEM, a complete loss of JAML surface expression was observed on the PMNs that had completed TEM. ${ }^{11}$ Thus, although PMNs migrating across epithelium can cause epithelial injury via secretion of soluble mediators, PMNs that reach luminal spaces may serve other purposes, namely, transduction of intracellular signaling via engagement of luminal epithelial surface receptors. Indeed, in contrast to a recent study that reported PMNs in migration inhibit epithelial wound closure via the release of soluble JAML, ${ }^{11}$ we demonstrated that adhesion of PMNs that have completed migration across IECs and are depleted of JAML and other harmful mediators triggered beneficial effects on epithelial cell proliferation and wound healing.

Activation has been also shown to delay PMN apoptosis. ${ }^{25,44,45}$ Although PMNs are short lived in the circulation, their life span is significantly increased as they enter inflamed tissues. $^{46,47}$ Consistent with this, we demonstrated that fMLF-activation of PMNs significantly delayed their apoptosis. 
Inhibition of apoptosis was further enhanced following TEM, consistent with increased PMN activation. Importantly, PMNs that remained bound to the apical epithelial surface after completing TEM were further protected from apoptosis. No further activation, assessed by changes in the expression of CD11b, was associated with PMN adhesion to epithelium following TEM, suggesting that PMN interactions with epithelial membrane receptors could provide additional anti-apoptotic cues. These findings provide evidence that, under conditions of intestinal inflammation, PMNs migrating into luminal spaces may remain bound to the epithelium for extended periods of time. These findings are particularly important in IBD, where PMN accumulation in luminal mucosal spaces can potentially contribute to wound healing and epithelial barrier restitution.

We further report that, once in the lumen, PMNs can bind to ICAM-1, which is abundantly expressed on the apical membrane of inflamed epithelial cells. ${ }^{29,48}$ This triggers activation of Akt and $\beta$-catenin signaling and results in enhanced epithelial cell proliferation.

Although soluble factors, such as epidermal growth factor and phosphoinosotide 3-kinase, were previously found to trigger Akt-dependent $\beta$-catenin transactivation, ${ }^{22,49}$ we identified a membrane-bound, PMN-adhesion ligand, ICAM-1, that can perform a similar function. Whether ICAM-1 can directly activate Akt or requires recruitment of adapter proteins is not clear; however, evidence from earlier work supports both of these mechanisms. For example, a known adapter protein, Ezrin, that is recruited downstream of ICAM-1, regulates ICAM-1-dependent signaling and has also been shown to activate Akt. ${ }^{50}$ Alternatively, another epithelial adhesion receptor, $\mathrm{N}$-cadherin, has also been shown to directly regulate $\beta$-catenin signaling. ${ }^{51}$

Intriguingly, elevated levels of cytokines, including IFN $\gamma$, that drive the inflammatory responses in mucosal tissues have been shown to suppress cell proliferation ${ }^{23}$ and negatively affect epithelial wound healing. ${ }^{28}$ IFN $\gamma$ treatment also induces expression of epithelial ICAM-1 (ref. 18) and alters epithelial barrier function, ${ }^{18,52,53}$ thus facilitating both increased PMN recruitment and enhanced PMN retention at the apical membrane. Our findings suggest that, under conditions of inflammation, PMN binding to ICAM-1 may counter the negative effects of IFN $\gamma$ and promote cell proliferation and wound healing. This was clearly demonstrated in experiments where specific $\mathrm{Ab} / \mathrm{PMN}$-mediated engagement of ICAM- 1 was able to suppress the IFN $\gamma$-dependent inhibition of wound healing.

Finally, we demonstrated that ICAM-1 has an important role in mucosal wound healing in-vivo. Our results showed that, in the absence of functional ICAM-1 in colonic mucosal wounds, intestinal epithelial Akt and $\beta$-catenin signaling was impaired. Consistent with the role of $\beta$-catenin in IEC cell proliferation, decreased numbers of proliferating crypt epithelial cells at wound edges were observed. ICAM-1 in endothelial cells has been previously shown to have low constitutive signaling activity; however, ICAM-1 signaling was greatly amplified upon dimerization and clustering. ${ }^{19,54}$ As PMN adhesion leads

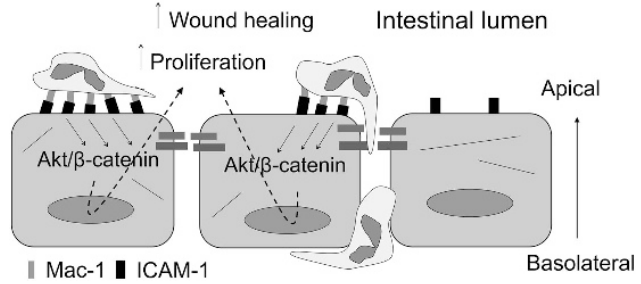

Figure 8 Polymorphonuclear neutrophil (PMN) migration into intestinal lumens and ligation of intercellular adhesion molecule-1 (ICAM-1) initiate reparative responses in epithelial cells. PMNs migrating across epithelial layers are retained at the luminal surface through binding to ICAM- 1 that is upregulated at the apical epithelial membrane when inflammation is present. Subsequently, PMN binding to ICAM-1 results in activation of Akt and $\beta$-catenin signaling leading to enhanced epithelial cell proliferation and wound healing.

to significant clustering of ICAM- $1,{ }^{54,55}$ it is not surprising that adherent PMNs could also amplify ICAM-1-dependent signaling events. Moreover, PMNs bound to apical epithelial membranes following TEM have been previously shown to exhibit ICAM-1-dependent locomotion, ${ }^{18}$ which likely facilitates further clustering and downstream signaling from epithelial ICAM-1.

The role for ICAM-1 in leukocyte migration across vascular endothelium is well established; and, in the absence of functional ICAM-1, leukocyte transendothelial migration is significantly delayed. ${ }^{54,56}$ Decreased leukocyte recruitment from the blood vessels into wounded tissues in ICAM-1ko animals may have contributed to delayed mucosal healing. However, even in the absence of ICAM-1, CD11b-positive cells were able to infiltrate wound beds following biopsy-based mucosal injury. Furthermore, Ab-mediated ICAM-1 engagement by injection directly into mucosal wounds in vivo also resulted in enhanced wound healing. Collectively, these findings suggest epithelial ICAM-1 signaling has a role in regulating wound healing in the intestinal lumen.

In summary, the findings in this study challenge the prevailing view that PMNs are exclusively pathologic in intestinal inflammation by demonstrating that the binding of post-migrated PMNs to epithelial-expressed ICAM-1 has beneficial effects on mucosal homeostasis and wound healing. ICAM-1 engagement and clustering by luminally adherent PMNs and subsequent activation of Akt and $\beta$-catenin signaling are illustrated in Figure 8. Our findings suggest that suppression of PMN recruitment to tissues as an exclusive anti-inflammatory treatment may not be the optimal approach because it may lead to aberrant epithelial function. Our data further suggest that the timing and context of PMN recruitment or suppression should be considered when designing antiinflammatory therapies. Finally, our findings support using epithelial ICAM-1 as a potential target for therapeutic approaches directed at improved mucosal healing.

\section{METHODS}

Animals. Male WT (C57BL6J; Jackson Laboratories, ME) and ICAM-1 knockout (ICAM-1ko) mice (B6.129S4-Icam1tm1Jcgr/J; Jackson Laboratories) between 12 and 15 weeks old were maintained under 
specific pathogen-free conditions at the Division of Animal Resources facility, Emory University.

Cells. Human T84 IECs were grown in Dulbecco's modified Eagle's medium (DMEM)-F12 50:50 (Sigma, St Louis, MO) with supplements as previously described. ${ }^{57} \mathrm{CHO}-\mathrm{K} 1$ and SW480 cells (used for transfection and TopFlash assays) were passaged in DMEM containing high glucose and supplemented with $10 \%$ fetal bovine serum, $100 \mathrm{U} \mathrm{ml}^{-1}$ penicillin, $100 \mu \mathrm{g} \mathrm{ml}^{-1}$ streptomycin, $10 \mathrm{mM}$ HEPES (pH 7.4), 2 mM L-glutamine, and 1\% non-essential amino acids. PMNs were isolated from human blood that was drawn from healthy volunteers and handled according to protocols for the protection of human subjects, as approved by the Emory University Hospital Institutional Review Board. PMNs were isolated by density gradient centrifugation ${ }^{57,58}$ and were used in experiments within $2 \mathrm{~h}$ of isolation.

Abs and reagents. Function blocking anti-human ICAM-1 (15.2) from Serotec (Raleigh, NC), anti-mouse ICAM-1mAb (YN1/1.7.4), anti-Ly-6G conjugated to Alexa 488, and anti-mouse MHC Class-I (W6/32) from eBioscience (San Diego, CA), anti-CD11b/CD18 mAb (CBRM1/29) purified in-house as previously described, ${ }^{59}$ Rabbit antiAkt and anti- $\beta$-catenin phospho forms from Cell Signaling (Danvers, MA), anti-Mouse-Alexa488 (Carlsbad, CA), anti-human MHC Class-I (1.B.548), and Rabbit anti-Ki67 from Abcam (Cambridge, MA), Isotype control IgG1 from BD Biosciences (San Jose, CA), horseradish peroxidase-conjugated anti-mouse and anti-rabbit IgGs from Jackson Immunoresearch (West Grove, PA). Click-iT EdU Alexa Fluor 488 Imaging Kit, molecular probes (Eugene, OR), Akt inhibitor VIII (EMD Millipore, Billerica, MA), 2,2' -azinobis-3-ethylbenzothiazoline6-sulfonic acid, L-mimosine, Hanks' Balanced Salt Solution (HBSS) with $\mathrm{Ca}^{2+}$ and $\mathrm{Mg}^{2+}(\mathrm{HBSS}+)$ and HBSS without $\mathrm{Ca}^{2+}$ and $\mathrm{Mg}^{2+}$ (HBSS -), DMEM including media supplements as well as chemotactic peptide fMLF, mouse IFN $\gamma$, and TNF $\alpha$ from Sigma.

ICAM-1 cross-linking. Ab-cross-linking protocols were performed as previously described (ref). Briefly, primary anti-ICAM-1, MHC Class1 , and CD55 $\left(20 \mu \mathrm{g} \mathrm{ml}^{-1}, 1 \mathrm{~h}\right)$ followed by secondary cross-linking Abs $\left(20 \mu \mathrm{g} \mathrm{ml}^{-1}, 60 \mathrm{~min}\right)$ were added apically to control/IFN $\gamma$ preexposed or scratch-wounded epithelial monolayers. In the case of $\mathrm{PMN}$-induced cross-linking, $2.5 \times 10^{5} \mathrm{PMN}$ per well (in $\mathrm{H}+, 1 \mathrm{~h}$, $37^{\circ} \mathrm{C}$ ) were added at a ratio of approximately one PMN to one IEC. Where specified, IECs were preincubated with Akt inhibitor $(10 \mu \mathrm{M})$ for $30 \mathrm{~min}$ at $37^{\circ} \mathrm{C}$. Unless otherwise specified, ICAM-1 expression was induced by treatment of the IECs with IFN $\gamma\left(100 \mathrm{U} \mathrm{ml}^{-1}, 24 \mathrm{~h}\right)$.

Western blot. Cell monolayers were lysed in RIPA lysis buffer ( $150 \mathrm{~mm}$ $\mathrm{NaCl}, 1 \% \mathrm{NP}-40,0.5 \%$ deoxycholic acid, $0.1 \%$ SDS, $50 \mathrm{~mm}$ Tris $\mathrm{pH} 8.0$ ) containing protease and phosphatase inhibitors (Sigma), sonicated, and cleared by centrifugation. Equal amounts of protein, determined using a bicinchoninic acid protein assay, were boiled in SDS sample buffer, separated by SDS-PAGE, and transferred onto nitrocellulose membranes. Membranes were blocked for $1 \mathrm{~h}$ with $5 \%$ (wt/vol) Roche (Basel, Switzerland) blocking reagent (Roche) in Tris-buffered saline and incubated with appropriate primary Abs overnight at $4{ }^{\circ} \mathrm{C}$, followed by secondary horseradish peroxidase-conjugated Abs. In the case of PMN adhesion to IECs, monolayers were washed three times with HBSS + to remove PMNs before protein extraction. By this method, $\sim 80 \%$ of adherent PMNs were removed (remaining adherent PMNs were counted in 10 randomly selected fields in 3 independent experiments, with three technical replicates for each experiment, using light microscopy). Murine colonic mucosal wounded and non-wounded tissues were collected using disposable biopsy punches of $2 \mathrm{~mm}$ radius and subjected to protocols similar to those described above for cell monolayers.

Flow cytometry. Adherent epithelial cells were collected (using Trypsin-EDTA, Sigma), washed, and stained for ICAM-1 (15.2 FITC, $\left.5 \mu \mathrm{g} \mathrm{ml}^{-1}\right)$. PMNs before and after TEM were stained for Mac-1 (ICRF44, $20 \mu \mathrm{l}$ per test). For PMN apoptosis assays, PMNs were either left in suspension (RPMI containing $5 \% \mathrm{BSA}, 37^{\circ} \mathrm{C}$ ), with or without fMLF activation, or were induced to migrate across collagen-coated filters or intestinal epithelial T84 cells, with or without subsequent adhesion to IFN $\gamma$-treated $\left(100 \mathrm{U} \mathrm{ml}^{-1}, 24 \mathrm{~h}\right)$ IECs in the presence of fMLF $(100 \mathrm{~nm})$. For both collagen-coated transfilters and IEC migration experiments, post-migrated PMNs were collected following $3 \mathrm{~h}$ migration, a time in which $\sim 90 \%$ of applied PMNs were observed to have completed migration (Supplementary Figure S1b). After $10 \mathrm{~h}$ incubation, PMNs were stained with Anexin- $\mathrm{V}$ and propidium iodide. PMNs were distinguished from epithelial cells by CD18/CD11b staining. All cell samples were analyzed using FACS Calibur and FlowJo software (Brea, CA).

Immunofluorescence labeling. Cultured cell monolayers were ethanol fixed, blocked with 5\% BSA in PBS, and incubated with the relevant primary $\mathrm{Ab}\left(10 \mu \mathrm{g} \mathrm{ml}^{-1}\right.$, over night at $\left.4{ }^{\circ} \mathrm{C}\right)$, either directly conjugated or followed by an appropriate fluorescently labeled secondary $\mathrm{Ab}$ ( $1 \mathrm{~h}$ at room temperature). For mouse tissue, punch biopsies of mucosal wounds and the corresponding non-wounded regions were frozen in optimum cutting temperature solution, cryosectioned $(7 \mu \mathrm{m}$ width), and fixed and stained as described for cell monolayers. All images were captured using an LSM 510 confocal microscope (Carl Zeiss Microimaging, Thornwood, NY) with panNeofluar $\times 20$ oil objective.

PMN adhesion assay. PMNs $\left(2.5 \times 10^{5}\right.$ cells per well in $\mathrm{H}+, 1 \mathrm{~h}$, $37^{\circ} \mathrm{C}$ ) were incubated with confluent T84 IEC monolayers that were pre-treated with IFN $\gamma\left(100 \mathrm{U} \mathrm{ml}^{-1}\right)$ at various times as specified. After three consecutive washes, adherent PMNs were quantified in 10 randomly selected fields of view three separate times for each experimental condition. ${ }^{18}$

PMN migration assay. PMN migration $\left(1 \times 10^{6}\right.$ cells per well in $\mathrm{HBSS}+, 1 \mathrm{~h}, 37^{\circ} \mathrm{C}$ ) across collagen-coated filters or epithelial monolayers in the physiologically relevant, basolateral-to-apical direction was induced with the introduction of fMLF gradient (100 nM) in low-adhesion plates (Corning, NY). Post-migrated PMNs were collected and immediately used in indicated experiments.

TOP/FOP-Flash transfection and luciferase assay. To assess the transcriptional activity of $\beta$-catenin following PMN or Ab-mediated ligation of ICAM-1, SW480 colonic epithelial cells were seeded in 48well tissue culture plates $(60,000$ cells per well) and transiently transfected with a $\beta$-catenin reporter containing $3 \mathrm{TCF} / \mathrm{LEF}-$ binding sites upstream of the luciferase reporter (TOP-Flash plasmid) or a negative control, FOP-Flash, which contains three TCF/LEF-mutated binding sites upstream of the luciferase reporter (Upstate Biotechnology, Lake Placid, NY). TOP/FOP plasmids were co-transfected with CMV-Renilla at a ratio 1:1,000. Transfections were carried out using Lipofectamine 2000 (Invitrogen Life Technologies, Grand Island, NY). Six hours after transfection, cell media were replaced with fresh DMEM supplemented with $10 \%$ serum. Seventy-two hours following transfection, PMN or ICAM-1 cross-linking protocols were applied for an additional $2 \mathrm{~h}$; and Luciferase and Renilla activity were measured in cell lysates using the Dual Luciferase Reporter Assay System (Promega) in the GloMax 96 Luminometer. Luciferase values were normalized to Renilla luciferase activity, and all experiments were performed three times.

Cell proliferation. Proliferation of cultured IECs following PMN- or Ab-ligation of ICAM-1 in epithelial cells in colonic crypts following mucosal injury was assessed by EdU incorporation using a Click-iT EdU Alexa 488 cell proliferation kit (Invitrogen, Grand Island, NY). For in vitro analysis, IECs were incubated with either post-migrated PMNs or anti-ICAM- 1 cross-linking Abs $\left(2 \mathrm{~h}, 37^{\circ} \mathrm{C}\right)$ before addition of EdU. At least five random fields per each condition were analyzed in three independent experiments, with three technical replicates for each experiment; the data are presented as percent of total cells proliferating in the field. For in vivo analysis, at days 2 and 4 post injury, EdU $(100 \mu \mathrm{g}$ 
per mouse) was administered via IP injection to WT and ICAM-1ko mice. Two hours later, mice were killed, colons extracted, and punch biopsies of wounded and non-wounded regions processed for immunofluorescence analysis. Data are presented as number of proliferating cells per crypt within $100 \mu \mathrm{m}$ from wounds.

Wound-healing assay. For in vitro experiments, standard scratchwound assays were employed. ${ }^{11}$ T84 IECs were grown to confluence in 24-well tissue culture plates. Linear, mechanical scratch wounds were introduced to each well using a $20-\mu l$ plastic pipette tip attached to low suction. Monolayers were washed with PBS to remove cellular debris and placed in complete media with the addition of an appropriate treatment. For biochemical experiments, multiple wounds were generated to enrich for migrating and proliferating cells. The rate of cell migration into scratch wounds was measured by determining the surface area devoid of epithelial cells immediately after wounding $(t=0)$ and at subsequent intervals as indicated. For in vivo experiments, a colonoscopic-biopsy-wound model was used as previously described. ${ }^{11,34}$ A high-resolution colonoscope (Coloview Veterinary Endoscope, Karl Stortz) was used to biopsy-injure the colon (three sites along the dorsal aspect) and monitor wound closure (up to 4 days) in anesthetized (ketamine $100 \mathrm{mg} \mathrm{kg}^{-1}$ and xylazine $5 \mathrm{mg} \mathrm{kg}^{-1}$ ) mice. At indicated intervals, mucosal wounds were harvested and prepared for immunofluorescence and western blot analysis. For immunecomplex injections, the complexes were prepared by incubating primary anti-ICAM-1 (YN1/1.7.4) or control rat IgG2a Abs with anti-Fc Abs at 1:2 ratio overnight. The complexes were injected directly into the wound area using a colonoscopy-based microinjection technique. $^{34}$

Statistics. Statistical significance was assessed by Student's $t$-test or by one-way analysis of variance with a Newman-Keuls Multiple Comparison Test using Graphpad Prism (V4.0, GraphPad Software, Inc., La Jolla, CA). Statistical significance was set at $P<0.05$. Unless otherwise specified all numerical data represent mean values from three to five independently performed experiments ( $N=3-5$ independent experiments). For experiments with technical replicates, the mean value of all replicates for each condition was first calculated, and these values were then averaged across independent experiments. All data presented as means of values \pm standard error mean.

SUPPLEMENTARY MATERIAL is linked to the online version of the paper at http://www.nature.com/mi

\section{ACKNOWLEDGMENTS}

We thank Emory University's Digestive Disease Research and Development Center core facility for culturing intestinal epithelial cell lines (supported by National Institutes of Health $[\mathrm{NIH}]$ DK064399). This work was supported in part by grants from the NIH (DK072564, DK061379, DK079392 to CP, and DK055679, DK059888 to A.N.), CDA from the Crohn's and Colitis Foundation of America to J.C.B., and NIH K01 DK101675 to R.S.

\section{DISCLOSURE}

The authors declared no conflict of interest.

c 2016 Society for Mucosal Immunology

\section{REFERENCES}

1. Koch, S. et al. The Wnt antagonist Dkk1 regulates intestinal epithelial homeostasis and wound repair. Gastroenterology 141, 259-268 (2011).

2. Sumagin, R., Robin, A.Z., Nusrat, A. \& Parkos, C.A. Activation of PKCbetall by PMA facilitates enhanced epithelial wound repair through increased cell spreading and migration. PLOS ONE 8, e55775 (2013).

3. Nava, P. et al. Interferon-gamma regulates intestinal epithelial homeostasis through converging beta-catenin signaling pathways. Immunity 32 , 392-402 (2010).
4. Brand, S. et al. IL-22 is increased in active Crohn's disease and promotes proinflammatory gene expression and intestinal epithelial cell migration. Am. J. Physiol. Gastrointest. Liver Physiol. 290, G827-G838 (2006).

5. Nava, P. et al. JAM-A regulates epithelial proliferation through Akt/betacatenin signalling. EMBO Rep. 12, 314-320 (2011).

6. Xavier, R.J. \& Podolsky, D.K. Unravelling the pathogenesis of inflammatory bowel disease. Nature 448, 427-434 (2007).

7. Laroux, F.S., Pavlick, K.P., Wolf, R.E. \& Grisham, M.B. Dysregulation of intestinal mucosal immunity: implications in inflammatory bowel disease. News Physiol. Sci. 16, 272-277 (2001).

8. Nusrat, A., Parkos, C.A., Liang, T.W., Carnes, D.K. \& Madara, J.L. Neutrophil migration across model intestinal epithelia: monolayer disruption and subsequent events in epithelial repair. Gastroenterology 113, 1489-1500 (1997).

9. Chin, A.C. \& Parkos, C.A. Neutrophil transepithelial migration and epithelial barrier function in IBD: potential targets for inhibiting neutrophil trafficking. Ann. N. Y. Acad. Sci. 1072, 276-287 (2006).

10. Ginzberg, H.H. et al. Neutrophil-mediated epithelial injury during transmigration: role of elastase. Am. J. Physiol. Gastrointest. Liver Physiol. 281, G705-G717 (2001).

11. Weber, D.A. et al. Neutrophil-derived JAML inhibits repair of intestinal epithelial injury during acute inflammation. Mucosal Immunol. 7, 1221-1232 (2014).

12. Sylvia, C.J. The role of neutrophil apoptosis in influencing tissue repair. J. Wound Care 12, 13-16 (2003).

13. Serhan, C.N. et al. Resolution of inflammation: state of the art, definitions and terms. FASEB J. 21, 325-332 (2007).

14. Zemans, R.L. et al. Neutrophil transmigration triggers repair of the lung epithelium via beta-catenin signaling. Proc. Natl Acad. Sci. USA 108, 15990-15995 (2011).

15. Zemans, R.L. et al. Role of beta-catenin-regulated CCN matricellular proteins in epithelial repair after inflammatory lung injury. Am. J. Physiol. Lung Cell Mol. Physiol. 304, L415-L427 (2013).

16. Lawrence, D.W. et al. Antiadhesive role of apical decay-accelerating factor (CD55) in human neutrophil transmigration across mucosal epithelia. J. Exp. Med. 198, 999-1010 (2003).

17. Brazil, J.C., Lee, W.Y., Kolegraff, K.N., Nusrat, A., Parkos, C.A. \& Louis, N.A. Neutrophil migration across intestinal epithelium: evidence for a role of CD44 in regulating detachment of migrating cells from the luminal surface. J. Immunol. 185, 7026-7036 (2010).

18. Sumagin, R., Robin, A.Z., Nusrat, A. \& Parkos, C.A. Transmigrated neutrophils in the intestinal lumen engage ICAM-1 to regulate the epithelial barrier and neutrophil recruitment. Mucosal Immunol. 7, 905-915 (2014).

19. Sumagin, R., Lomakina, E. \& Sarelius, I.H. Leukocyte-endothelial cell interactions are linked to vascular permeability via ICAM-1-mediated signaling. Am. J. Physiol. Heart Circ. Physiol. 295, H969-H977 (2008).

20. Lawson, C. \& Wolf, S. ICAM-1 signaling in endothelial cells. Pharmacol. Rep. 61, 22-32 (2009).

21. Liu, G. et al. ICAM-1-activated Src and eNOS signaling increase endothelial cell surface PECAM-1 adhesivity and neutrophil transmigration. Blood 120, 1942-1952 (2012).

22. Fang, D. et al. Phosphorylation of beta-catenin by AKT promotes betacatenin transcriptional activity. J. Biol. Chem. 282, 11221-11229 (2007).

23. Nava, P. et al. IFNgamma-induced suppression of beta-catenin signaling: evidence for roles of Akt and 14.3.3zeta. Mol. Biol. Cell 25, 2894-2904 (2014).

24. Rubel, C., Fernandez, G.C., Dran, G., Bompadre, M.B., Isturiz, M.A. \& Palermo, M.S. Fibrinogen promotes neutrophil activation and delays apoptosis. J. Immunol. 166, 2002-2010 (2001).

25. Coxon, A., Tang, T. \& Mayadas, T.N. Cytokine-activated endothelial cells delay neutrophil apoptosis in vitro and in vivo. A role for granulocyte/ macrophage colony-stimulating factor. J. Exp. Med. 190, 923-934 (1999).

26. Velnar, T., Bailey, T. \& Smrkolj, V. The wound healing process: an overview of the cellular and molecular mechanisms. J. Int. Med. Res. 37, 1528-1542 (2009).

27. Sturm, A. \& Dignass, A.U. Epithelial restitution and wound healing in inflammatory bowel disease. World J. Gastroenterol. 14, 348-353 (2008).

28. Tong, Q. et al. Interferon-gamma inhibits T84 epithelial cell migration by redirecting transcytosis of beta1 integrin from the migrating leading edge. J. Immunol. 175, 4030-4038 (2005). 
29. Kaiserlian, D., Rigal, D., Abello, J. \& Revillard, J.P. Expression, function and regulation of the intercellular adhesion molecule-1 (ICAM-1) on human intestinal epithelial cell lines. Eur. J. Immunol. 21, 2415-2421 (1991).

30. Hentzen, E.R. et al. Sequential binding of CD11a/CD18 and CD11b/CD18 defines neutrophil capture and stable adhesion to intercellular adhesion molecule-1. Blood 95, 911-920 (2000).

31. Sumagin, R., Prizant, H., Lomakina, E., Waugh, R.E. \& Sarelius, I.H. LFA-1 and Mac-1 define characteristically different intralumenal crawling and emigration patterns for monocytes and neutrophils in situ. J. Immunol. 185, 7057-7066 (2010).

32. Parkos, C.A., Delp, C., Arnaout, M.A. \& Madara, J.L. Neutrophil migration across a cultured intestinal epithelium. Dependence on a CD11b/CD18mediated event and enhanced efficiency in physiological direction. J. Clin. Invest. 88, 1605-1612 (1991).

33. Wang, G., Miskimins, R. \& Miskimins, W.K. Mimosine arrests cells in G1 by enhancing the levels of p27(Kip1). Exp. Cell Res. 254, 64-71 (2000).

34. Leoni, G. et al. Annexin A1-containing extracellular vesicles and polymeric nanoparticles promote epithelial wound repair. J. Clin. Invest. 125, 12151227 (2015).

35. Leoni, G. et al. Human neutrophil formyl peptide receptor phosphorylation and the mucosal inflammatory response. J. Leukoc. Biol. 97, 87-101 (2015).

36. Smith, J.A. Neutrophils, host defense, and inflammation: a double-edged sword. J. Leukoc. Biol. 56, 672-686 (1994).

37. Rigby, K.M. \& DeLeo, F.R. Neutrophils in innate host defense against Staphylococcus aureus infections. Semin. Immunopathol. 34, 237-259 (2012).

38. Chin, A.C., Lee, W.Y., Nusrat, A., Vergnolle, N. \& Parkos, C.A. Neutrophilmediated activation of epithelial protease-activated receptors-1 and -2 regulates barrier function and transepithelial migration. J. Immunol. 181, 5702-5710 (2008).

39. Shimoda, N., Fukazawa, N., Nonomura, K. \& Fairchild, R.L. Cathepsin g is required for sustained inflammation and tissue injury after reperfusion of ischemic kidneys. Am. J. Pathol. 170, 930-940 (2007).

40. Davey, A., McAuley, D.F. \& O'Kane, C.M. Matrix metalloproteinases in acute lung injury: mediators of injury and drivers of repair. Eur. Respir. J. 38 , 959-970 (2011).

41. Medina, C. et al. Matrix metalloproteinase-9 modulates intestinal injury in rats with transmural colitis. J. Leukoc. Biol. 79, 954-962 (2006).

42. Lacy, P. Mechanisms of degranulation in neutrophils. Allergy Asthma Clin. Immunol. 2, 98-108 (2006).

43. Zemans, R.L., Colgan, S.P. \& Downey, G.P. Transepithelial migration of neutrophils: mechanisms and implications for acute lung injury. Am. J. Respir. Cell Mol. Biol. 40, 519-535 (2009).

44. Biffl, W.L., Moore, E.E., Moore, F.A. \& Barnett, C.C. Jr. Interleukin-6 delays neutrophil apoptosis via a mechanism involving platelet-activating factor. J. Trauma 40, 575-578 (1996). Discussion 8-9.

45. Klein, J.B. et al. Granulocyte-macrophage colony-stimulating factor delays neutrophil constitutive apoptosis through phosphoinositide 3-kinase and extracellular signal-regulated kinase pathways. J. Immunol. 164, 4286-4291 (2000).

46. Kolaczkowska, E. \& Kubes, P. Neutrophil recruitment and function in health and inflammation. Nat. Rev. Immunol. 13, 159-175 (2013).

47. Yoo, S.K. \& Huttenlocher, A. Spatiotemporal photolabeling of neutrophil trafficking during inflammation in live zebrafish. J. Leukoc. Biol. 89, 661-667 (2011).

48. Parkos, C.A. et al. Expression and polarization of intercellular adhesion molecule-1 on human intestinal epithelia: consequences for CD11b/ CD18-mediated interactions with neutrophils. Mol. Med. 2, 489-505 (1996).

49. Lee, G. et al. Phosphoinositide 3-kinase signaling mediates beta-catenin activation in intestinal epithelial stem and progenitor cells in colitis. Gastroenterology 139, 869-881 (2010).

50. Hiscox, S. \& Jiang, W.G. Ezrin regulates cell-cell and cell-matrix adhesion, a possible role with E-cadherin/beta-catenin. J. Cell Sci. 112 (Pt 18), 3081-3090 (1999).

51. Zhang, J., Shemezis, J.R., McQuinn, E.R., Wang, J., Sverdlov, M. \& Chenn, A. AKT activation by $\mathrm{N}$-cadherin regulates beta-catenin signaling and neuronal differentiation during cortical development. Neural Dev. 8, 7 (2013).

52. Watson, C.J., Hoare, C.J., Garrod, D.R., Carlson, G.L. \& Warhurst, G. Interferon-gamma selectively increases epithelial permeability to large molecules by activating different populations of paracellular pores. J. Cell Sci. 118, 5221-5230 (2005).

53. Boivin, M.A., Roy, P.K., Bradley, A., Kennedy, J.C., Rihani, T. \& Ma, T.Y. Mechanism of interferon-gamma-induced increase in T84 intestinal epithelial tight junction. J. Interferon Cytokine Res. 29, 45-54 (2009).

54. Yang, L. et al. Endothelial cell cortactin coordinates intercellular adhesion molecule-1 clustering and actin cytoskeleton remodeling during polymorphonuclear leukocyte adhesion and transmigration. J. Immunol. 177, 6440-6449 (2006).

55. Yang, L., Froio, R.M., Sciuto, T.E., Dvorak, A.M., Alon, R. \& Luscinskas, F.W. ICAM-1 regulates neutrophil adhesion and transcellular migration of TNF-alpha-activated vascular endothelium under flow. Blood 106, 584-592 (2005).

56. Sumagin, R. \& Sarelius, I.H. A role for ICAM-1 in maintenance of leukocyteendothelial cell rolling interactions in inflamed arterioles. Am. J. Physiol. Heart Circ. Physiol. 293, H2786-H2798 (2007).

57. Parkos, C.A., Colgan, S.P., Delp, C., Arnaout, M.A. \& Madara, J.L. Neutrophil migration across a cultured epithelial monolayer elicits a biphasic resistance response representing sequential effects on transcellular and paracellular pathways. J. Cell Biol. 117, 757-764 (1992).

58. Chin, A.C., Fournier, B., Peatman, E.J., Reaves, T.A., Lee, W.Y. \& Parkos, C.A. CD47 and TLR-2 cross-talk regulates neutrophil transmigration. J. Immunol. 183, 5957-5963 (2009).

59. Balsam, L.B., Liang, T.W. \& Parkos, C.A. Functional mapping of CD11b/ CD18 epitopes important in neutrophil-epithelial interactions: a central role of the I domain. J. Immunol. 160, 5058-5065 (1998). 